\title{
Población doctoral graduada en Costa Rica. Contraste con datos globales y posibles incidencias
}

Recibido: 20 de noviembre 2019

Revisado: 6 de marzo 2020

Aprobado: 9 de mayo 2020

\section{Mario Alejandro Morúa}

Saborío

Costarricense. Grado de Doctorado (Ph. D) en Ciencias de la

Administración, por la Universidad Estatal a

Distancia de Costa Rica .

Investigador del Programa de Investigación en

Fundamentos de Educación a Distancia (PROIFED) de la UNED, Costa Rica, y coordinador de la Comisión

Técnica de Investigación y

Posgrado del Sistema

Regional Centroamericano y del Caribe de Investigación y Posgrado (SIRCIP-CSUCA),

Costa Rica.

Correo electrónico: mmorua@uned.ac.cr

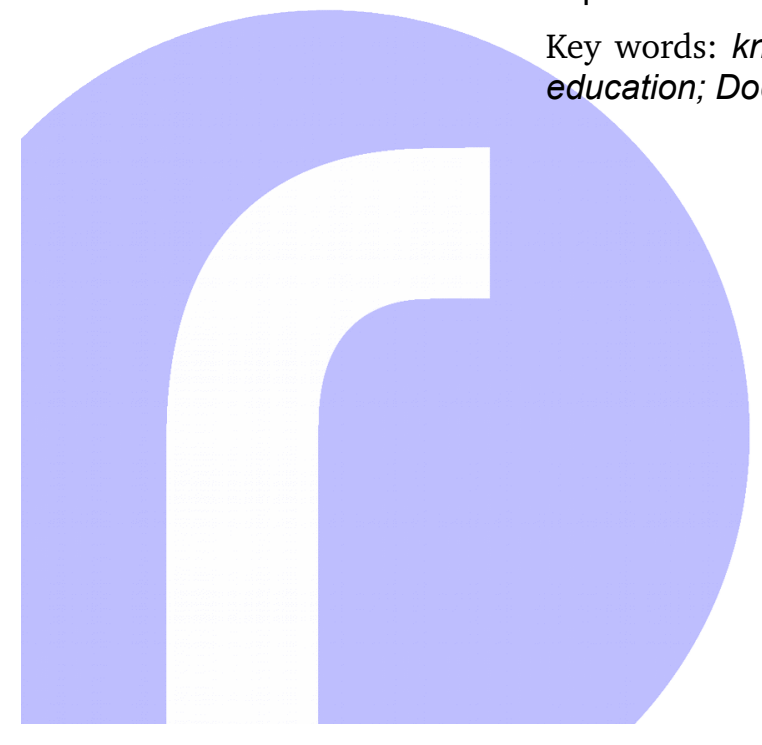

https://revistas.uned.ac.cr/index.php/rupturas (c) (1) (2)
Resumen: En este artículo, se expondrán datos e información correspondientes a los 15 países que, en el mundo, graduaron más personas con el grado de doctor/a académico/a en el año 2014, así como la descripción de algunas relaciones con el índice de desarrollo humano (IDH), con el índice de competitividad global (ICG) y con los índices demográficos, a partir de exploración teórica, documental y el análisis descriptivo que de esta se deriva. Dichos elementos se contrastarán con los respectivos datos e información perteneciente a Costa Rica, como un caso de referencia en Latinoamérica por la magnitud de su gasto (inversión), en el rubro de "Educación" en general y "Educación superior" en particular.

Palabras clave: conocimiento; desarrollo humano; desarrollo de la educación; educación superior; doctorado

\section{Doctoral Population Graduated in Costa Rica. Contrast with Global Data and Possible Incidents}

Abstract: This article will present data and information corresponding to the 15 countries that in the world graduated more people with the degree of academic doctor in 2014, as well as the description of some relationships with the respective indices of: human development (HDI), global competitiveness (ICG) and demographics; this, from theoretical exploration, documentary, and the descriptive analysis that is derived from it. The foregoing will be contrasted with the respective data and information belonging to Costa Rica, as a reference case in Latin America for the magnitude of its expenditure (investment), in the heading of "Education" in general and "Higher Education" in particular.

Key words: knowledge; human development; education development; higher education; Doctorate 


\section{Introducción}

La educación superior universitaria, tal y como fue concebida en Europa a partir del siglo XI, con la histórica y ancestral influencia de las universidades árabes, ha sido el principal referente en América para la formación superior universitaria desde el establecimiento de las colonias en el Nuevo Mundo. Esta evolucionó y se consolidó en los siglos subsiguientes; ha tenido -y continúa teniendo-grandes y profundas transformaciones, las cuales han hecho que, en la actualidad, sus propósitos, esfuerzos y recursos sean orientados hacia la profesionalización del talento humano, de tal manera que, a este, entre otras muchas cosas, se le facilite -aunque no siempre con éxito- su inserción en la población económicamente activa de sus respectivos contextos.

El hecho de generar conocimiento como función sustantiva de la educación superior universitaria, a partir de la actividad investigativa, así como el pensamiento crítico y creativo (masa crítica), ha tenido que contrastarse con la marcada tendencia a priorizar la adquisición y el perfeccionamiento de competencias y habilidades, orientadas más hacia la aplicación inmediata del conocimiento, que a la propia generación de este. Con algunas excepciones, como se expondrá más adelante, podrían no estar generando mayor impacto o, al menos, incidencia en indicadores de desarrollo humano y competitividad, aunque sí un desbalance en algunas relaciones; por ejemplo: costo/beneficio y eficiencia/eficacia, principalmente por el altísimo gasto (inversión) requerido en general por la educación superior pública costarricense y, en particular, por los programas doctorales que ofrece.

Tal tendencia es notoria, en especial, en el predominio de diseños y ofertas académicas de carreras y programas formativos que priorizan la adquisición y el perfeccionamiento de competencias y habilidades, orientadas más hacia la aplicación inmediata del conocimiento, que a la propia generación. Y los posgrados no escapan a tal tendencia, sobre todo, aquellos denominados "maestrías profesionales". ¿Cuál es la problemática derivada de esta tendencia? Un marcado y progresivo faltante, en las universidades públicas costarricenses, de talento humano graduado con algún doctorado académico, y que, a la vez, mantenga responsabilidades académicas, a saber: investigación, docencia y acción social. Es decir, cada vez faltan más doctoras y doctores académicos en las universidades públicas costarricenses con responsabilidades orientadas a la generación, gestión y transferencia de conocimiento.

Contextos con mayor desarrollo que el de los países latinoamericanos, como el Espacio Europeo de Educación Superior (EEES), privilegian, con el ejercicio de responsabilidades académicas en cualquiera de sus expresiones, a aquellas poblaciones que exclusivamente hayan obtenido su doctorado académico. España y Alemania representan tan solo dos casos concretos, en los cuales solamente personas graduadas de algún programa doctoral pueden ser profesores para impartir docencia y, a la vez, ser responsables titulares para el desarrollo de programas y de proyectos de investigación. 
¿Existen brechas y asimetrías del ejemplo anterior con respecto a Costa Rica? Posiblemente muchas. Pero, ineludiblemente, bajo esas condiciones, sumadas a otros factores estructurales y coyunturales, a España le fue posible salir de su crítica situación fiscal y económica, muy parecida a la costarricense; y, a Alemania, consolidar su liderazgo en Europa, como una de las economías con mayor crecimiento per cápita, similar al caso de Costa Rica en la región.

Es muy probable que esa realidad alemana le permita a este país enfrentar, con solvencia, las crisis que recientemente emergen en su contexto inmediato, relacionadas con su desarrollo humano y competitividad; esto, a la vez, representa una brecha con respecto a Costa Rica.

Aparte de los variados y diversos campos disciplinares del saber, amplia y profundamente abordados por la educación superior universitaria, las nomenclaturas en los diplomas y títulos que esta otorga, así como su respectiva clasificación y ubicación en pregrado, grado y posgrado, han privilegiado la especialización y la profesionalización, según sus respectivos niveles de profundidad, alcance, rigor académico y complejidad, entre otros descriptores, en función de la mencionada adquisición y el perfeccionamiento de competencias y de habilidades.

La generación de conocimiento como producto esencial de la actividad intelectual y académica, además de la transferencia, del fomento y del ejercicio del pensamiento crítico y creativo, como asidero teórico-conceptual indispensable tanto para el perfeccionamiento de competencias y de habilidades como para la generación de nuevas destrezas, han logrado mantener su histórico, natural e inherente arraigo en el más elevado grado académico que la educación superior universitaria pueda otorgar a una persona: el doctorado académico. Luego de una rigurosa formación académica y científica en los programas conducentes a ese grado, estos mantienen su énfasis y enfoque en la investigación, al menos, desde la concepción y el diseño de los programas que conducen dichos doctorados académicos. Así sería esto, salvo que la realidad de la comunidad científica y académica mundial cambiara en los últimos días.

Tal y como recién se señaló, en la actualidad, se percibe, en las universidades latinoamericanas -por supuesto, Costa Rica no es la excepción-, un déficit de talento humano que ostente el grado máximo de doctorado académico en los diferentes campos disciplinares del saber. Esto representa no solo un reto social en materia de formación superior, sino también un desafío urgente de atender por parte de las casas de estudios superiores estatales, como principales instancias que, tradicional e históricamente, han tenido el llamado expreso para generar, preservar y transferir el conocimiento por medio de la investigación, de la docencia y de la acción social, con el consecuente gasto (inversión) en talento humano y recursos tangibles e intangibles.

De perpetuarse e incrementarse tal déficit, se comprometería la continuidad y la sostenibilidad de los esfuerzos orientados al desarrollo científico y académico logrado en cada universidad de Latinoamérica , frente a las vertiginosas 
transformaciones sociales y científicas, que constantemente se traducen, según Pablo González Casanova (2005, 11), en "los descubrimientos que se iniciaron con la cibernética, la epistemología genética, la computación, los sistemas autorregulados, adaptativos y autopoiéticos, las ciencias de la comunicación, las ciencias de la organización, las del caos determinista, los atractores y los fractales"; ya que, se limitaría la generación, la actualización y la transferencia oportuna del conocimiento que se fomenta y se promueve, de manera natural, desde los programas doctorales, así como de las investigaciones de alto nivel que se generan posteriormente por parte de las poblaciones graduadas de dichos programas.

Ninguna de las economías con mayores niveles de desarrollo en el mundo podría afirmar, hoy por hoy, que sus aciertos e identificación de oportunidades para mejorar, en términos de desarrollo y competitividad, se encuentran divorciadas del gasto (inversión) que históricamente hicieran tanto en materia de educación en general; y, específicamente, en formación superior universitaria. Incluso, en esta última, la inversión en investigación, innovación y desarrollo; y de acuerdo con el contexto compartido anteriormente por González Casanova $(2005,11)$, no es este el momento para propiciar tal divorcio. Sin embargo, existe una tendencia a la reducción de los presupuestos asignados a esos rubros, tal y como se muestra en la siguiente figura 1 :

\section{Figura 1. La baja en el gasto público en I+D y los riesgos proteccionistas pueden representar una amenaza para la innovación, declara la OCDE.}

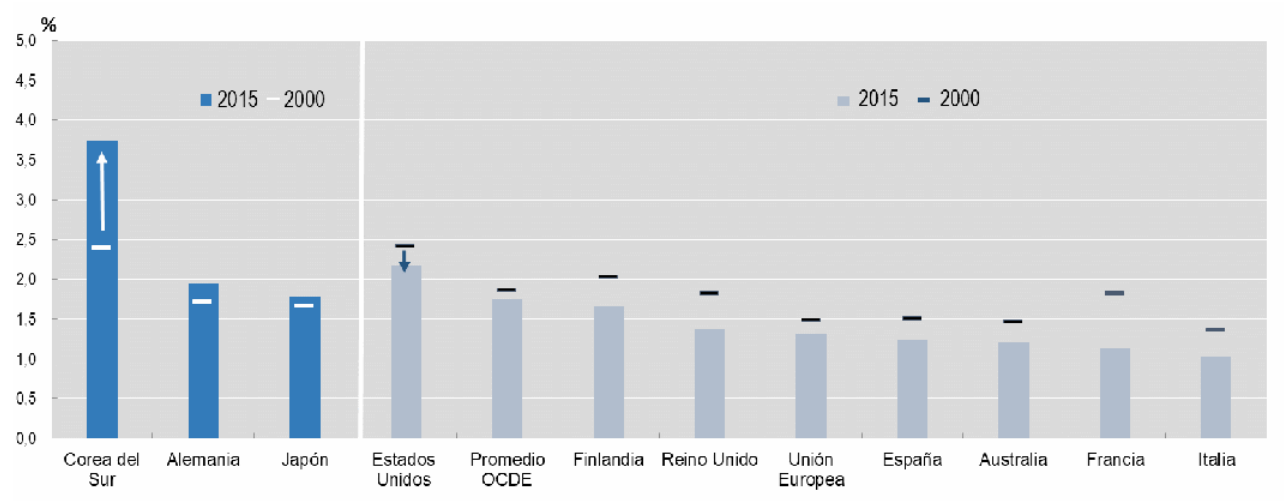

Organización para la Cooperación y el Desarrollo Económico (OCDE, 2016).

Este artículo conforma una reseña de literatura y de material ya publicados. Sin embargo, se enfoca en relacionar datos e información sobre población universitaria, población doctoral graduada, así como datos demográficos, de desarrollo humano y de competitividad, de los 15 países que, en el mundo, graduaron más personas con doctorado académico durante el 2014. Posteriormente, estos se contrastarán con esos mismos datos e información de la realidad costarricense, por ser esta, según la Organización para la Coopera- 
ción y el Desarrollo Económico (OCDE), un caso de referencia en Latinoamérica, al considerar que su "gasto público en educación superior como un porcentaje del Producto Interno Bruto (PIB), es superior al de la mayoría de los países miembros de ese organismo" $(2017,18)$.

Con respecto al monto del gasto público costarricense en educación superior, al que se hace referencia en el párrafo anterior, Javier Córdoba (2019) menciona, en un artículo del Semanario Universidad, que "El presupuesto de las universidades públicas para el próximo año quedó fijado en $\phi 512.781,51 \mathrm{mi}-$ llones y para la Universidad Técnica Nacional el monto será de $\phi 35.677,88$ millones"; lo que equivale, aproximadamente, a un gasto (inversión) total que supera los 900 millones de dólares ${ }^{1}$.

Dichos datos e información sobre los cuales se fundamenta la presente exposición se obtuvieron de publicaciones realizadas a partir del año 2014 por instancias, como el Foro Económico Mundial (FEM), el Banco Mundial (BM), el Programa de las Naciones Unidas para el Desarrollo (PNUD), el Consejo Nacional de Rectores en Costa Rica (CONARE) y el Programa Estado de la $\mathrm{Na}$ ción. Así, se guarda la esperanza de que lo reseñado pueda contextualizarse y extrapolarse a otras realidades, con el propósito de sugerir acciones que permitan, no solo reflexionar al respecto, sino también afrontar la realidad que se compartirá, de tal manera que sea posible dirigir el cambio, de manera disruptiva y sostenible en el tiempo, más que de adaptarse a este; lo anterior, se hará desde perspectivas de investigación más amplias y profundas derivadas y enriquecidas por el pensamiento complejo, las ciencias complejas, las ciencias de la organización, el pensamiento sistémico y la indagación sistémica.

Aun cuando en esta publicación se recurre a descripciones cuantitativas de frecuencias para apoyar la exposición de su contenido, su propósito no es fundamentar su exposición en la representatividad cuantitativa, sino más bien generar espacios para la discusión, el diálogo y la formulación de hipótesis y tesis, a partir de los cuales sea posible tomar decisiones, pero a la vez, profundizar en el tema con otras pesquisas de carácter explicativo, propositivo y evaluativo.

Además, el método aplicado para su elaboración (hermeneútico-dialéctico) hace que las características del presente documento sean coherentes y consistentes con el enfoque humanístico-cualitativo, "el cual tiene un interés práctico por comprender la problemática descrita, sin que eso limite esos espacios de discusión y el diálogo permanente desde un enfoque sociocrítico con interés emancipador y el propósito de transformar dicha problemática" (Villalobos, 2017, 15-16).
1. Los montos de la nota periodística son consistentes con datos e información disponibles sobre este tema en el sitio web oficial del Consejo Nacional de Rectores (CONARE). 


\section{DESARROLLO}

\section{Método, metodología y técnicas}

En cuanto al método, como se mencionó, se siguió el hermenéutico-dialéctico, el cual, según Mario Alejandro Morúa Saborío $(2016,95)$ que cita a Alicia Gurdian Fernández (2007, 147-149):

es un método general de comprensión y la interpretación es el modo natural de conocer de los seres humanos. La hermenéutica tiene como misión descubrir los significados de las cosas, interpretar lo mejor posible las palabras, los escritos, los textos y los gestos, así como cualquier acto y obra, pero conservando su singularidad en el contexto del que forma parte. De acuerdo con la lógica dialéctica, las partes son comprendidas desde el punto de vista del todo. Dilthey (1900) llamó circulo hermenéutico a este proceso interpretativo, es decir al movimiento que va del todo a las partes y de las partes al todo tratando de buscarle sentido.

Con respecto a la metodología seguida en la elaboración de la presente exposición, entendida como la ruta o secuencia de actividades que conducen a resultados concretos, conclusiones y recomendaciones, una vez analizados dichos resultados, se hizo la exploración teórica y documental, así como la observación participante y entrevista con persona experta. Esto condujo hacia análisis descriptivos de los diferentes datos e información recopilados, con los cuales se exponen síntesis analíticas relacionadas con la problemática planteada. Los datos e información fueron recopilados por medio de las siguientes técnicas: a) revisión teórica y documental disponible en medios impresos, electrónicos y publicados en sitios especializados, b) minutas en la observación participante y c) entrevista no estructurada con persona experta.

\section{Elementos teóricos considerados}

En este apartado, convendría iniciar haciendo referencia a los cinco conceptos expuestos como palabras clave del presente documento: conocimiento, desarrollo humano, desarrollo de la educación, educación superior, doctorado, ya que, el significado de cada uno conforma el hilo conductor que, más adelante, derivará en conclusiones y en recomendaciones. Para estos efectos, y con el fin de disponer de conceptos más libres de sesgos técnicos, profesionales o epistémicos, así como con mayor aceptación global y local, se 
recurrirá a las dos siguientes fuentes en internet: a) el Tesauro de la Unesco y b) La Real Academia Española (RAE).

Conocimiento: "Conjunto de informaciones y saberes ligados a un contexto particular, compartidos por los miembros de una comunidad y necesarios para la realización de una actividad determinada" (Tesauro, 2019).

Desarrollo humano: "Proceso de ampliación de las opciones del individuo, tanto en lo relativo al desarrollo de las capacidades humanas (mejora de la salud, profundización en el conocimiento) como en el uso de estas capacidades" (Tesauro, 2019).

Desarrollo de la educación: "Evolución positiva de una o más características del sistema educativo en función de ciertos criterios de apreciación” (Tesauro, 2019).

Educación superior: "Etapa de la enseñanza que sucede al nivel secundario, sea cual sea la duración del curso o el certificado obtenido" (Tesauro, 2019).

Doctorado: "Conocimiento acabado y pleno en alguna materia" (RAE, 2019).

Para los efectos de esta exposición y de la misma formación superior en el sistema universitario público costarricense, en este punto, resulta necesario aclarar la diferencia que existe entre los doctorados profesionales que, por costumbre, se les han otorgado a las personas que concluyeron con el grado académico de licenciatura en el campo de las ciencias médicas o alguna otra disciplina del campo de la salud y los doctorados académicos, entendidos los segundos como el mayor de los posgrados ofrecidos en dicho sistema. Claro está, sin que esto sea un argumento que desacredite o subvalore a los doctorados profesionales, son, simplemente, diferentes.

Con base en la nomenclatura de grados y de títulos que establece el Consejo Nacional de Rectores en Costa Rica (CONARE), la siguiente es la definición para el doctorado académico:

Es el grado máximo que otorga la Educación Superior Universitaria Estatal. Su propósito fundamental es el de formar investigadores académicos. El énfasis se establece en el rigor y la profundidad con que se hace la investigación y los cursos deben ser solo un apoyo a esta actividad. El plan de estudios debe ser flexible y eficaz en desplazar claramente la carga académica del estudiante hacia las tareas de investigación. El programa deberá promover pasantías en el extranjero para sus estudiantes, así como otras experiencias académicas internacionales. 
Este nivel académico requiere el dominio instrumental de al menos una segunda lengua.

El doctorado académico se caracteriza por los siguientes elementos:

Créditos: Mínimo 50, máximo 70 adicionales a la maestría. Estos créditos incluyen los asignados al trabajo de tesis.

Duración: Mínimo 4 ciclos lectivos de 15 semanas o su equivalente sobre la maestría.

(Reglamento General Estudiantil de la Universidad Estatal a Distancia en Costa Rica, artículo 2, inciso f), 2012, 56).

\begin{abstract}
La exposición en este texto, en todo momento, mantendrá su enfoque en los doctorados académicos; por lo tanto, y con el fin de ampliar lo referente al concepto de doctorado, más allá de su concepción normativa, significado semántico y, en concordancia con los propósitos de la educación superior, se transcribe textualmente, a continuación, la tabla con los descriptores y los respectivos resultados de aprendizaje esperados para el nivel de doctorado, según el Marco de Cualificaciones para la Educación Superior Centroamericana $(2013,20)$ :
\end{abstract}


Tabla 1. Descriptores y resultados de aprendizaje esperados para el nivel doctoral

\begin{tabular}{|c|c|}
\hline Descriptor & Resultados de aprendizaje esperados \\
\hline $\begin{array}{l}\text { Saberes disciplinarios } \\
\text { y profesionales }\end{array}$ & $\begin{array}{l}\text { Posee un conocimiento amplio y profundo de la teoría y práctica de } \\
\text { una o varias disciplinas, y el dominio de las habilidades y métodos de } \\
\text { investigación que le permiten aportar a la solución de problemas en } \\
\text { diferentes contextos y generar nuevos conocimientos reconocidos } \\
\text { nacional e Internacionalmente. }\end{array}$ \\
\hline $\begin{array}{l}\text { Aplicación de } \\
\text { conocimientos, } \\
\text { análisis de } \\
\text { información y } \\
\text { resolución de } \\
\text { problemas e } \\
\text { innovación }\end{array}$ & $\begin{array}{l}\text { Identifica problemas complejos no resueltos, para los cuales los } \\
\text { marcos teóricos actuales no tienen respuesta, propone y sustenta } \\
\text { explicaciones y planteamientos teóricos, metodológicos o } \\
\text { tecnológicos alternativos a los existentes. Analiza, contrasta y evalúa } \\
\text { de forma crítica las teorías, metodologías y procedimientos que se } \\
\text { producen en su campo. } \\
\text { Diseña, gestiona, desarrolla planes, programas y proyectos de } \\
\text { investigación, así como colaboraciones científicas en contextos } \\
\text { multidisciplinarios e interdisciplinarios, nacionales e internacionales. } \\
\text { Realiza contribuciones originales y significativas a la investigación } \\
\text { científica a través de la generación del nuevo conocimiento, que } \\
\text { amplían las fronteras del saber en su ámbito, reconocidas por la } \\
\text { comunidad científica nacional e internacional. }\end{array}$ \\
\hline $\begin{array}{l}\text { Autonomía con } \\
\text { responsabilidad } \\
\text { personal, profesional } \\
\text { y social }\end{array}$ & $\begin{array}{l}\text { Ejerce su profesión en forma responsable dentro de los marcos } \\
\text { normativos y éticos de su campo profesional, y reflexiona sobre la } \\
\text { incidencia de sus decisiones en los aspectos humanos, sociales y } \\
\text { ambientales. Asume una actitud de aprendizaje permanente y lo } \\
\text { promueve en su equipo de trabajo. Conforma y participa en } \\
\text { comunidades científicas institucionales e interinstitucionales y } \\
\text { colabora en la formación de nuevos científicos. Comparte sus } \\
\text { conocimientos, experiencias y aprendizajes, asimismo promueve su } \\
\text { uso para la búsqueda de nuevas opciones de solución a problemas } \\
\text { de su entorno para la generación de política pública. Reflexiona sobre } \\
\text { su experiencia en la actividad investigadora y la comparte para } \\
\text { fortalecer las comunidades de aprendizaje y orientar la opinión } \\
\text { pública. Participa y promueve, en contextos académicos y } \\
\text { profesionales, la innovación, la transferencia del conocimiento y el } \\
\text { avance científico tecnológico, social y cultural. }\end{array}$ \\
\hline Comunicación & $\begin{array}{l}\text { Participa en discusiones (científicas académicas) nacionales e } \\
\text { internacionales, y divulga los resultados de su actividad investigativa } \\
\text { a través de ponencias, revistas académicas o científicas indexadas y } \\
\text { en sitios web, tanto en su lengua oficial como en una segunda lengua. } \\
\text { Promueve sus aportes ante la comunidad científica nacional e } \\
\text { internacional, mediante la publicación referenciada o con el registro } \\
\text { de derecho intelectual. }\end{array}$ \\
\hline $\begin{array}{l}\text { Interacción } \\
\text { profesional, cultural y } \\
\text { social }\end{array}$ & $\begin{array}{l}\text { Gestiona y lidera equipos y proyectos de investigación innovadores y } \\
\text { de colaboración científica, nacionales o internacionales, en contextos } \\
\text { interdisciplinarios o transdisciplinarios, multiculturales o de } \\
\text { transferencia de conocimiento. Asume el liderazgo con visión } \\
\text { estratégica en investigaciones interdisciplinarias. Participa } \\
\text { proactivamente en comunidades científicas en contextos } \\
\text { interdisciplinarios de generación y de transferencia de conocimiento. }\end{array}$ \\
\hline
\end{tabular}

Marco de Cualificaciones para la Educación Superior Centroamericana (2013). 
Con base en los elementos teóricos anteriormente considerados y el propósito de describir posibles relaciones que trasciendan el ámbito científico y académico, desde una perspectiva más concreta y pragmática, que, además, respalde los argumentos orientados a fomentar la formación de personas con doctorado académico, se considerarán en esta publicación, los siguientes dos índices mundialmente aceptados como referentes de desarrollo y competitividad: el índice de desarrollo humano (IDH) y el índice de competitividad global (ICG), como una expresión concreta del conocimiento científico y académico generado.

Cada uno de esos dos índices está compuesto por componentes que expresan, no solo la implementación de políticas, de regulaciones y de acciones orientadas al desarrollo humano y a la competitividad, sino también la concreción tanto del quehacer científico y académico, en todos sus niveles, como la transferencia del conocimiento que se genera y aplica a partir de dicho quehacer en los diferentes sectores productivos y económicos.

De acuerdo con el el Programa de las Naciones Unidas para el Desarrollo (PNUD) $(2018,3)$, el IDH lo integran los componentes de a) Expectativa de vida, b) Educación y c) Producto interno bruto (PIB). Se mide en una escala de 0 a 1 , dentro de la cual, se presenta la siguiente clasificación: menos de $0,550=$ desarrollo humano bajo; entre 0,550 y $0,699=$ desarrollo humano medio; entre 0,700 y $0,799=$ desarrollo humano alto; y 0,800 o más $=$ desarrollo humano muy alto.

En otras palabras, el IDH estrecha correlaciones directas entre los factores asociados al sector de la salud (expectativa de vida); la generación y distribución de riqueza (PIB); y, expresamente para los efectos de esta publicación, la educación, en todos sus niveles y ámbitos de acción, como inductor de movilidad social.

Por otra parte, según el FEM (2014, 9, 13), "el ICG lo componen: a) Requerimientos básicos, b) Potenciadores de eficiencia y c) Factores de innovación y sofisticación, y se mide en una escala de $1-7$ ". De acuerdo con los informes del FEM, publicados entre los años 2014 y 2017, la economía suiza ocupó el primer lugar del ICG en el mundo, obteniendo valores entre 5,70 y 5,80; para el caso de Costa Rica en esos mismos periodos, los valores se ubicaron entre 4,42 y 4,41 , ocupando las posiciones 51 y 52 , respectivamente.

Cada uno de los tres componentes que integran el ICG, se encuentran incorporados, a su vez, por pilares de diferente naturaleza; entre dichos pilares y para los efectos de esta publicación, se hace mención a los siguientes, estrechamente correlacionados con el IDH y con el tema central de esta exposición: "a) Requerimientos básicos - Pilar 1: Instituciones, b) Potenciadores de eficiencia - Pilar 5: Educación superior y capacitación, y c) Factores de innovación y sofisticación - Pilar 12: Innovación" (FEM, 2014, 9).

Con respecto a las relaciones costo/beneficio y eficiencia/eficacia, mencionadas en el apartado de la "Introducción", según Mario Alejandro Morúa Saborío (2016, 56), al citar a Luis Eduardo González Fiegehen y Hernán Ayarza Elorza $(1996,19)$, la relación "costo / beneficio involucra comparar costos y 
retornos. También implica la exploración de costos alternativos". Esto, para efectos de tener claridad en cuanto al gasto o inversión que se hace y los beneficios esperados, que, en principio, deberían ser proporcionales, al menos, con el gasto o inversión realizada, lo que necesariamente implica responsabilidad y transparencia en el manejo presupuestario. En cuanto a la relación eficiencia/eficacia, según también Morúa $(2016,56)$ :

esta relación se refiere básicamente a la capacidad para disponer tanto del talento humano, como también de ciertos recursos (tangibles e intangibles) para el logro de uno o varios fines específicos, y, a la vez, la capacidad de lograr esos fines con ese talento humano y recursos disponibles. En otras palabras, se refiere al balance que debe existir entre la capacidad para disponer de los factores que se necesitan para lograr algo y la capacidad de utilizar adecuadamente esos factores para lograrlo.

\section{Resultados}

Según el Foro Económico Mundial (FEM), en el año 2014, de los 15 países en el mundo que más doctores y doctoras graduaron, Sudáfrica fue el que menos graduó: su población doctoral graduada, en ese año, fue de 2060 personas. Por el contrario, Estados Unidos de Norteamérica fue el país que más doctores y doctoras graduó en el año 2014, para un total de 67449 personas graduadas, tal y como se puede observar en la siguiente figura 2, en la cual también se muestran datos de los 13 países restantes incluidos en esta clasificación; dicho sea de paso, ninguno latinoamericano y once de esos quince países, actualmente, son miembros de la Organización para la Cooperación y el Desarrollo Económico (OCDE).

Al contrastar los datos de la figura 2 con los datos de la siguiente tabla 2, es evidente que la tasa de personas con doctorado académico por cada 100 000 habitantes mantiene un rango amplio que va de 1 a 39, lo que, a su vez, evidencia que, en ninguno de esos 15 países, la población doctoral graduada, en el año 2014 , igualó o superó el $1 \%$ de su respectiva población total censada en el año 2014 (cantidad total de habitantes), según datos del Banco Mundial. Sin embargo, en esos términos de relación entre población doctoral graduada y población global censada en el año 2014, el Reino Unido es el país que presentó una mayor relación (39 personas con doctorado acadé- 
mico por cada 100000 habitantes), mientras que Indonesia es el país que mantuvo una relación menor (1 por cada 100000 habitantes).

Figura 2. Países con más doctores y doctoras universitarios en el año 2014. Unidades expresadas en miles

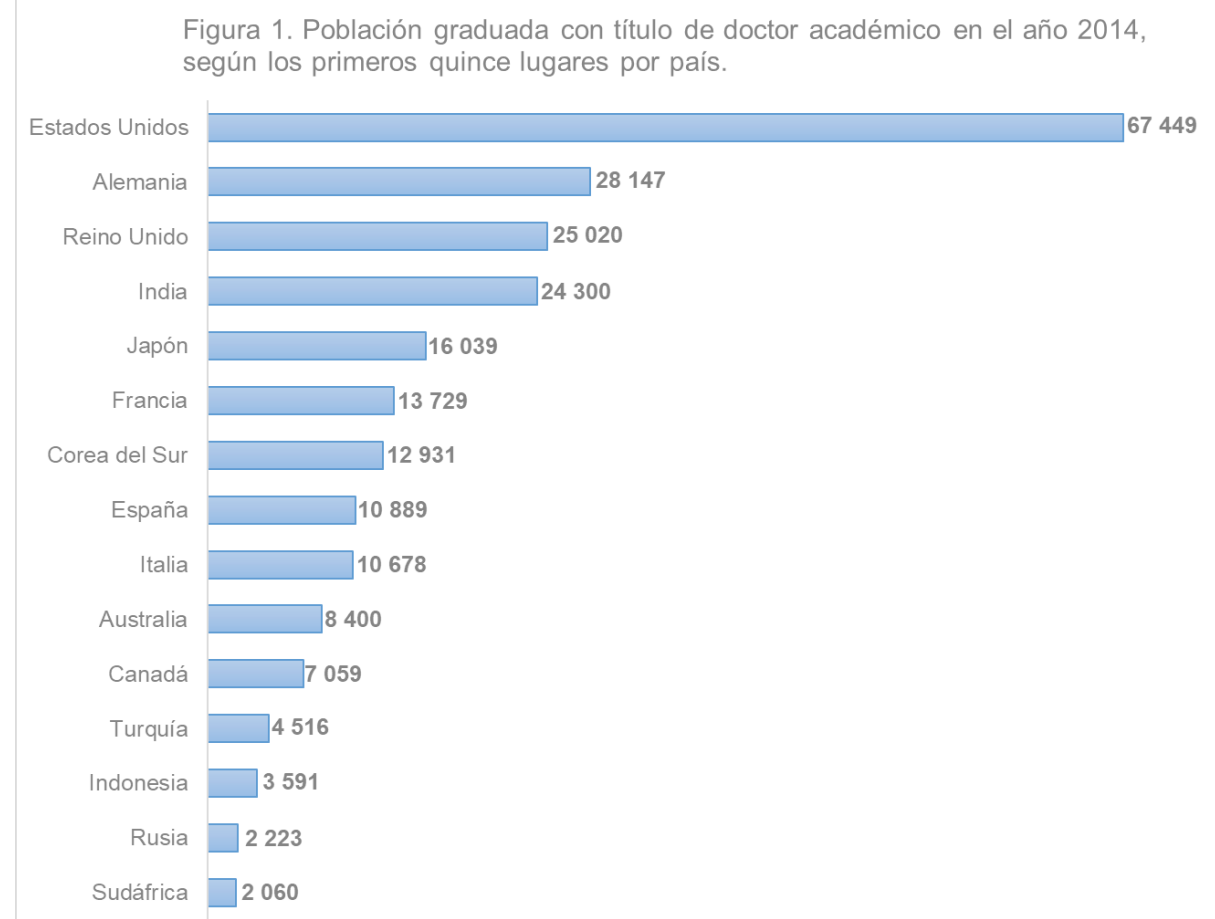

Foro Económico Mundial (FEM) (2017)

Ahora bien, tal y como se expondrá, esta simple relación entre población doctoral graduada y población total censada es insuficiente para inferir conclusiones debidamente fundamentadas sobre los aportes concretos de las poblaciones que obtienen el grado de doctor académico. Es más, pareciera que no hay una clara intención por parte de estos países, ni tan siquiera por llegar, al menos, al $1 \%$ de población doctoral graduada, respecto de la población total censada. 
Tabla 2. Población graduada de doctorados académicos y población total censada en el año 2014, según país.

\begin{tabular}{llccc}
\hline$N^{\circ}$ & \multicolumn{1}{c}{ País } & $\begin{array}{c}\text { Población doctoral } \\
\text { graduada año 2014 }\end{array}$ & $\begin{array}{c}\text { Población } \\
\text { censada año } \\
\mathbf{2 0 1 4}\end{array}$ & $\begin{array}{c}\text { Población } \\
\text { doctoral por cada } \\
\text { 100 mil habitantes }\end{array}$ \\
\hline 1 & Estados Unidos & 67449 & 318622525 & 21 \\
2 & Alemania & 28147 & 80982500 & 35 \\
3 & Reino Unido & 25020 & 64613160 & 39 \\
4 & India & 24300 & 1293859294 & 2 \\
5 & Japón & 16039 & 127276000 & 13 \\
6 & Francia & 13729 & 66316092 & 21 \\
7 & Corea del Sur & 12931 & 50746659 & 25 \\
8 & España & 10889 & 46480882 & 23 \\
9 & Italia & 10678 & 60789140 & 18 \\
10 & Australia & 8400 & 23504138 & 36 \\
11 & Canadá & 7059 & 35535348 & 20 \\
12 & Turquía & 4516 & 77030628 & 6 \\
13 & Indonesia & 3591 & 255131116 & 1 \\
14 & Rusia & 2223 & 143819666 & 2 \\
15 & Sudáfrica & 2060 & 54539571 & 4 \\
\hline
\end{tabular}

Nota: El valor relativo porcentual (\%) corresponde a la relación entre población graduada de doctorados académicos y población total censada en el año 2014, según datos del Foro Económico Mundial (FEM) y el Banco Mundial (BM), respectivamente.

Elaboración propia con base en datos del Foro Económico Mundial (2017) y el Banco Mundial (2018).

En términos de generación de conocimiento con alto rigor científico y académico, es muy sencillo justificar la importancia del nivel doctoral en la educación superior universitaria. Es decir, en esos términos, expresar la importancia de disponer con más talento humano con doctorados académicos, sobre todo en la población de docentes, de investigadores e investigadoras nombrados o contratados por las universidades, es prácticamente una verdad de Perogrullo. Incluso, según el Sistema Nacional de Acreditación de la Educación Superior (SINAES), se trata de un dato que forma parte de los componentes, de los criterios y de las evidencias, dentro del Manual de acreditación oficial de programas de posgrados del Sistema Nacional de Acreditación de la Educación Superior de Costa Rica.

Según la Corporación de Radiodifusión Británica (BBC por sus siglas en inglés), por una parte, con base en datos e información de la OCDE "Canadá es el país con mayor porcentaje de personas universitarias en el mundo (56\%), entre los 23 y los 64 años de edad" (BBC, 2018). Por otra parte, la BBC también informa, con base en la misma fuente (OCDE), que "para el caso de Costa Rica se estima que el $23 \%$ de las personas entre 23 y 64 años de edad, realizó algún tipo de estudios superiores" (BBC, 2018). 
En virtud de estas últimas afirmaciones se ilustran, de alguna manera, dos cosas: la evidente brecha entre ambos países y, a la vez, una representación relativa de su población universitaria dentro de un rango de edad con alta productividad intelectual, con respecto a la población total de cada país. No obstante, en ninguno de los dos casos, se podrían traducir sus respectivos porcentajes, en una mayor cantidad de personas universitarias graduadas con algún grado o posgrado académico. Por lo expuesto en las líneas precedecentes, a continuación, se describirán relaciones entre los datos de la figura 2 y la tabla 2, con el índice de desarrollo humano (IDH) y el índice de competitividad global (ICG).

Con respecto a cada uno de los componentes que integran ambos índices, el de "Educación" -en sus correspondientes ámbitos de acción, a saber: educación general básica (primaria, secundaria) y técnica y superior universitaria con sus respectivos niveles (grado, pregrado y posgrado)- mantiene relación y una expectativa de incidencia directa con cada uno de los demás componentes que integran cada índice. Por lo tanto, las variaciones en dichos componentes y el respectivo índice como tal, necesariamente, se generan en uno o más de los ámbitos de acción anteriormente mencionados para el componente "Educación", ya sea por injerencia o acción directa, transversal o ambas, en las respectivas poblaciones económicamente activas.

Tabla 3. Índice de Desarrollo Humano del año 2014 al 2017, según los 15 países con mayor población graduada con título de doctorla y población censada al año 2014, respectivamente.

\begin{tabular}{llccccccc}
\hline$N^{\circ}$ & \multicolumn{1}{c}{ País } & $\begin{array}{c}\text { Población } \\
\text { doctoral } \\
\text { graduada } \\
\text { año 2014 }\end{array}$ & $\begin{array}{c}\text { Población } \\
\text { censada año } \\
\mathbf{2 0 1 4}\end{array}$ & $\begin{array}{c}\text { IDH } \\
\mathbf{2 0 1 4}\end{array}$ & $\begin{array}{c}\text { IDH } \\
\mathbf{2 0 1 5}\end{array}$ & $\begin{array}{c}\text { IDH } \\
\mathbf{2 0 1 6}\end{array}$ & $\begin{array}{c}\text { IDH } \\
\mathbf{2 0 1 7}\end{array}$ & $\begin{array}{c}\text { Nivel } \\
\text { IDH }\end{array}$ \\
\hline 1 & Estados Unidos & 67449 & 318622525 & 0,918 & 0,92 & 0,922 & 0,924 & Muy alto \\
2 & Alemania & 28147 & 80982500 & 0,93 & 0,933 & 0,934 & 0,936 & Muy alto \\
3 & Reino Unido & 25020 & 64613160 & 0,919 & 0,918 & 0,92 & 0,922 & Muy alto \\
4 & India & 24300 & 1293859294 & 0,618 & 0,627 & 0,636 & 0,64 & Medio \\
5 & Japón & 16039 & 127276000 & 0,903 & 0,905 & 0,907 & 0,909 & Muy alto \\
6 & Francia & 13729 & 66316092 & 0,894 & 0,898 & 0,899 & 0,901 & Muy alto \\
7 & Corea del Sur & 12931 & 50746659 & 0,896 & 0,898 & 0,9 & 0,903 & Muy alto \\
8 & España & 10889 & 46480882 & 0,88 & 0,885 & 0,889 & 0,891 & Muy alto \\
9 & Italia & 10678 & 60789140 & 0,874 & 0,876 & 0,878 & 0,88 & Muy alto \\
10 & Australia & 8400 & 23504138 & 0,933 & 0,936 & 0,938 & 0,939 & Muy alto \\
11 & Canadá & 7059 & 35535348 & 0,918 & 0,92 & 0,922 & 0,926 & Muy alto \\
12 & Turquía & 4516 & 77030628 & 0,778 & 0,783 & 0,787 & 0,791 & Alto \\
13 & Indonesia & 3591 & 255131116 & 0,683 & 0,686 & 0,691 & 0,694 & Medio \\
14 & Rusia & 2223 & 143819666 & 0,807 & 0,813 & 0,815 & 0,816 & Muy alto \\
15 & Sudáfrica & 2060 & 54539571 & 0,685 & 0,692 & 0,696 & 0,699 & Medio \\
\hline
\end{tabular}

Elaboración propia con base en datos del Foro Económico Mundial (2017), el Banco Mundial (2018) y PNUD (2014-2017). 
En otras palabras, por las acciones formativas, informativas y de transferencia de conocimiento propias de la "educación", se genera y aplica el conocimiento, el cual, articulado con otros factores socioeconómicos, culturales, ambientales y políticos, explica, justifica y fundamenta las variaciones que experimenten cada uno de estos índices y sus componentes. A partir de tal explicación y fundamentación, se posibilita y se potencia la implementación de acciones orientadas a generar las condiciones desde las cuales sea posible aumentar o, al menos, mantener en el tiempo los valores en cada índice.

En las tablas 3 y 4, se exponen datos agrupados por país, según población graduada con título de doctor/a (FEM, 2017); población total censada (BM, 2018); IDH (PNUD, 2014-2017); e ICG (FEM, 2014-2017), con el propósito de contrastar y de hacer comparaciones entre esos datos, de tal manera que sea posible establecer relaciones significativas.

Tabla 4. Índice de competitividad global del año 2014 al 2017, según los 15 países con mayor población graduada con título de doctor/a y población censada al año 2014, respectivamente.

\begin{tabular}{llcccc}
\hline$N^{\circ}$ & \multicolumn{1}{c}{ País } & $\begin{array}{c}\text { Población } \\
\text { doctoral } \\
\text { graduada año }\end{array}$ & $\begin{array}{c}\text { Población } \\
\text { censada año }\end{array}$ & \multicolumn{2}{c}{ ICG } \\
\cline { 5 - 6 } & & $\mathbf{2 0 1 4}$ & $\mathbf{2 0 1 4}$ & $\mathbf{2 0 1 4 - 2 0 1 5}$ & $\mathbf{2 0 1 6 - 2 0 1 7}$ \\
\hline 1 & Estados Unidos & 67449 & 318622525 & 5,54 & 5,7 \\
2 & Alemania & 28147 & 80982500 & 5,49 & 5,57 \\
3 & Reino Unido & 25020 & 64613160 & 5,41 & 5,49 \\
4 & India & 24300 & 1293859294 & 4,21 & 4,52 \\
5 & Japón & 16039 & 127276000 & 5,47 & 5,48 \\
6 & Francia & 13729 & 66316092 & 5,08 & 5,2 \\
7 & Corea del Sur & 12931 & 50746659 & 4,96 & 5,03 \\
8 & España & 10889 & 46480882 & 4,55 & 4,68 \\
9 & Italia & 10678 & 60789140 & 4,42 & 4,5 \\
10 & Australia & 8400 & 23504138 & 5,16 & 5,19 \\
11 & Canadá & 7059 & 35535348 & 5,24 & 5,27 \\
12 & Turquía & 4516 & 77030628 & 4,46 & 4,39 \\
13 & Indonesia & 3591 & 255131116 & 4,57 & 4,52 \\
14 & Rusia & 2223 & 143819666 & 4,37 & 4,51 \\
15 & Sudáfrica & 2060 & 54539571 & 4,35 & 4,47 \\
\hline
\end{tabular}

Elaboración propia con base en datos del Fondo Económico Mundial (FEM) (2017), del Banco Mundial (2018). 
Al contrastar los datos e información de la figura 2 y de las tablas 2, 3 y 4, en términos generales, se infiere lo siguiente:

1. Con respecto al IDH, de los 15 países que se observan en la figura 2 anterior, 11 mantienen valores del IDH que los ubican en el nivel muy alto $(+0,800)$, 1 en el nivel alto (entre 0,700 y 0,799$)$ y 3 en el nivel medio (entre 0,550 y 0,699), ninguno en el nivel bajo de IDH. En otras palabras, $80 \%$ de los casos observados mantiene un rango alto o superior de IDH.

2. Se observa que las variaciones interanuales de IDH, reportadas entre los años 2014, 2015, 2016 y 2017, para cada uno de esos países, mantienen tendencia al alza, en los 15 casos. Por ende, se infiere participación activa de múltiples factores socioeconómicos, incluso las poblaciones graduadas con doctorados académicos, tanto en el origen de esos valores de IDH como en el sostenimiento en dicha tendencia.

3. En relación con los valores del ICG, en una escala de 1 a 7 , los 15 países de la figura 2, presentan valores altos ubicados entre 5,54 y 4,21 . A su vez, para cada uno de esos países, la variación interanual en este índice mantiene, también, tendencia al alza. Asimismo, se infiere participación activa de las poblaciones graduadas con doctorados académicos.

Ahora bien, en el caso específico de Costa Rica, el Quinto Informe sobre el Estado de la Educación $(2015,184)$ ofrece los siguientes datos sobre personas graduadas en el año 2014 con algún diploma de posgrado. Se trata de un dato global donde se incluyen: especialidades, maestrías (profesionales y académicas) y doctorados, tal y como se observa en la tabla 5.

Tabla 5. Cantidad de personas graduadas, total y con algún diploma de posgrado en Costa Rica, durante el año 2014, según universidades públicas y privadas.

\begin{tabular}{lccc}
\hline \multirow{2}{*}{ Universidad } & Graduación total & \multicolumn{2}{c}{ Posgrados } \\
\cline { 3 - 4 } & & $\%$ & Total \\
\hline U. Públicas & 14594 & 11,1 & 1620 \\
U. Privadas & 31753 & 10,8 & 3429 \\
Total & 46347 & & 5049 \\
\hline
\end{tabular}

Elaboración propia con base en datos obtenidos del capítulo IV del Quinto Informe del Estado de la Educación (2015).

En la tabla 5, se observa que las universidades públicas costarricenses graduaron en total 14594 personas; de las cuales, 11\% obtuvo algún diploma de posgrado (especialidad, maestrías y doctorados). Dicho estudio no detalla, por separado, la cantidad de personas graduadas con especialidades, maes- 
trías o doctorados, sino que hace una sola contabilización bajo la categoría de "Posgrados".

Según Diana Arce Flores et al $(2017,9)$, en el Estudio de seguimiento a personas graduadas de doctorado en las cuatro universidades estatales costarricenses periodo 2000-2011, indican que "en ese periodo de tiempo (11 años), se graduó en el nivel doctoral una población de 160 personas, proveniente de 11 programas de doctorado ofrecidos por las cuatro universidades estatales costarricenses", es decir, en 11 años, se graduó $10 \%$ del total de posgrados graduados en el año 2014 en las universidades públicas costarricense. Esto significa que, en promedio, cada año durante el periodo comprendido, entre los años 2000 y 2011, se graduaron 14,5 personas en alguno de los doctorados ofrecidos por las cuatro universidades estatales costarricenses, lo cual marca una baja tasa anual de graduación doctoral por parte de estas casas de estudios superiores, al menos para ese periodo.

Ninguno de los dos estudios recién mencionados: $V$ Informe del Estado de la Educación y el Estudio de seguimiento a personas graduadas de doctorado realizado en CONARE ofrecen elementos suficientes con base en los cuales se pueda, al menos, inferir la cantidad de personas graduadas en el nivel doctoral, entre los años 2011 y 2014, por parte de las universidades estatales o privadas. Tampoco se ofrecen, en dichos estudios, evidencias de variaciones contextuales, estructurales o coyunturales que, eventualmente, justifiquen una variación futura en la tendencia antes inferida sobre la cantidad de personas graduadas en programas doctorales.

Por lo tanto y con base en datos e información del Quinto Informe del Estado de la Educación y el Estudio de seguimiento a personas graduadas de doctorado, para los efectos de la presente publicación, una estimación aproximada y aceptable es que, para el año 2014 en Costa Rica, el sistema universitario estatal graduó un promedio anual de 14,5 personas en el nivel doctoral.

No obstante y con base en los datos e información recientemente expuestos en el Séptimo Informe Estado de la Educación, publicado el pasado mes de agosto del presente año y, según se puede inferir de la siguiente tabla 6 , entre el año 2014 y el 2016, la estimación del promedio anual, antes mencionada, de 14,5 personas graduadas con algún doctorado académico ofrecido por las universidades públicas costarricenses, evidentemente, tuvo que experimentar una reducción, ya que en dicho periodo, se entregaron menos diplomas de posgrados.

Tabla 6. Diplomas de posgrados otorgados por la educación superior costarricense

\begin{tabular}{llll}
\hline Posgrados & $\mathbf{2 0 0 5}$ & $\mathbf{2 0 1 0}$ & $\mathbf{2 0 1 6}$ \\
\hline U. Públicas & 1260 & 1589 & 1370 \\
U. Privadas & 1643 & 2971 & 3167 \\
Total & 2903 & 4560 & 4537 \\
\hline
\end{tabular}

Séptimo Informe del Estado de la Educación (2019). 
Por una parte, la magnitud del valor correspondiente a esa reducción es aún más incierta en esta ocasión, por cuanto se continúa agrupando los diplomas de posgrados, sin especificar los valores correspondientes al doctorado académico. Por otra parte, los estudios e informes, posteriores al año 2014, relacionados con este tema, continúan sin ofrecer evidencias debidamente fundamentadas sobre cambios 0 , al menos, ajustes estructurales orientados a transformar esta realidad.

En el caso particular de Costa Rica, al contrastar los valores relacionados con la estimación de la población doctoral graduada y la censada en el año 2014, así como los respectivos valores de IDH e ICG costarricenses, se observa que el país iguala a Turquía y supera a India, en los valores reportados para el IDH; con respecto al ICG 2014-2015, Costa Rica igualó a Italia y superó a India, a Rusia y a Sudáfrica. Mientras que, en el periodo 2016-2017, Costa Rica, únicamente, superó a Turquía, según los datos que se observa en la siguiente tabla 7:

Tabla 7. Comparativo de datos sobre población doctoral graduada, población censada, IDH e ICG para Costa Rica, Turquía, India, Italia, Rusia, Sudáfrica.

\begin{tabular}{|c|c|c|c|c|c|c|}
\hline Indicador & $\begin{array}{l}\text { Datos de } \\
\text { Costa Rica }\end{array}$ & $\begin{array}{l}\text { Datos de } \\
\text { Turquía }\end{array}$ & $\begin{array}{l}\text { Datos de } \\
\text { India }\end{array}$ & $\begin{array}{c}\text { Datos de } \\
\text { Italia }\end{array}$ & $\begin{array}{c}\text { Datos de } \\
\text { Rusia }\end{array}$ & $\begin{array}{l}\text { Datos de } \\
\text { Sudáfrica }\end{array}$ \\
\hline $\begin{array}{l}\text { Estimación } \\
\text { población } \\
\text { doctoral } \\
\text { graduada } \\
\text { año } 2014\end{array}$ & 14,5 & 4516 & 24300 & 10678 & 2223 & 2060 \\
\hline $\begin{array}{l}\text { Población } \\
\text { censada } \\
\text { año } 2014\end{array}$ & 4757575 & 77030628 & $\begin{array}{c}1293859 \\
294\end{array}$ & 60789140 & $\begin{array}{c}143819 \\
666\end{array}$ & 54539571 \\
\hline IDH 2014 & 0,78 & 0,778 & 0,618 & 0,874 & 0,807 & 0,685 \\
\hline IDH 2015 & 0,788 & 0,783 & 0,627 & 0,876 & 0,813 & 0,692 \\
\hline IDH 2016 & 0,791 & 0,787 & 0,636 & 0,878 & 0,815 & 0,696 \\
\hline IDH 2017 & 0,794 & 0,791 & 0,64 & 0,88 & 0,816 & 0,699 \\
\hline $\begin{array}{l}\text { ICG } \\
2014-2015\end{array}$ & 4,42 & 4,46 & 4,21 & 4,42 & 4,37 & 4,35 \\
\hline $\begin{array}{l}\text { ICG } \\
2016-2017\end{array}$ & 4,41 & 4,39 & 4,52 & 4,5 & 4,51 & 4,47 \\
\hline
\end{tabular}

Elaboración propia con base en datos del Fondo Económico Mundial (2017), del Banco Mundial (2018) y del Programa de las Naciones Unidas para el Desarrollo (2014-2017).

Para el caso de Costa Rica, con base en el contraste entre los datos de la tabla 7 y los datos de la figura 2 y las tablas de la 2 a la 5 , se observa que, al establecer la relación entre la estimación de la población doctoral graduada en el año 2014 y la población total censada, según datos del BM, tal relación, en términos relativos, no representa, al menos, un porcentaje aproximado a 
esta misma relación, pero estimada para los países mencionados en la figura 2. Esto podría interpretarse como una población cuya participación relativa, en términos de desarrollo humano y de competitividad o, al menos en alguno de sus componentes, tiene efecto diluido y limitado, a pesar del elevado gasto (inversión) que representa, para las casas de estudios superiores públicas costarricenses, graduar a una persona en alguno de los doctorados académicos ofrecidos.

Con base en datos también del Quinto Informe. Estado de la Educación, para el año 2014 en Costa Rica, se contabilizaron "53 universidades privadas autorizadas por el Consejo Nacional de Educación Superior (CONESUP)" $(2015,184)$. No todas las 53 universidades privadas ofrecen programas doctorales. Sin embargo, si hipotéticamente se extrapolara a cada una de las universidades privadas el dato inferido anteriormente de 14,5 personas graduadas con algún doctorado académico en el año 2014, se obtendría una estimación aproximada de 795 personas graduadas con algún doctorado académico en el año 2014, lo cual supondría un total de 810 personas graduadas con algún doctorado académico en ese año, entre universidades públicas y privadas costarricenses.

Si se asumiera como real tal suposición, la relación entre población graduada de doctorados académicos y la población total censada en el año 2014, para el caso de Costa Rica, eventualmente, sería comparable con la relación estimada para el caso de Italia $(0,018 \%)$, Canadá $(0,020 \%)$; incluso, superaría a Japón $(0,013 \%)$. No obstante, esto seguirá siendo un supuesto poco probable de extrapolar, ya que son pocas las universidades privadas que ofrecen programas doctorales.

En contraposición al análisis que se ha hecho con base en la figura 2 y tablas de la 2 a la 7, los valores de IDH ubican a Costa Rica en un nivel alto, lo mismo que sus valores de ICG. Esto genera una ruptura en el análisis que, hasta este punto, se ha realizado, por cuanto se rompe la relación o correspondencia entre los valores del IDH e ICG costarricenses, con respecto a los valores de estos mismos indicadores para los 15 países que más personas con título de doctor/a académico/a graduaron en el año 2014. Es decir, Costa Rica, con una reducida tendencia de población doctoral graduada por año, al menos por parte de la educación superior pública, mantiene valores de IDH e ICG comparables con los 15 países que graduaron más personas con título de doctor/a académico/a en el año 2014.

La pregunta que surge, entonces, es, para el caso de Costa Rica, ¿cómo se fundamentan sus indicadores de IDH e ICG si, en contraste con los países de la figura 2, la generación propia de conocimiento, a partir de programas doctorales y de las investigaciones que de estos se deriven, pareciera tener poca incidencia en el incremento y en el sostenimiento de dichos indicadores? 


\section{Conclusiones}

1. Los 15 países que más personas graduaron con doctorado académico, durante el año 2014, mantienen una proporción igual o mayor a 1 por cada 100000 habitantes, según la población total censada en el año 2014; es decir, al menos tienen una persona con doctorado académico por cada 100000 habitantes. Esa proporción se ubica entre 1 (Indonesia) y 39 (Reino Unido) (tabla 2). Para el caso de Costa Rica, tal relación es prácticamente nula en comparación con Indonesia y, aún más, con el Reino Unido.

2. Con respecto a los 15 países que en el mundo graduaron más personas con algún doctorado académico en el año 2014, se observan datos, a partir de los cuales, es posible inferir: a) correlaciones asociadas con la cantidad de personas que obtuvieron algún doctorado académico, b) los valores de IDH e ICG, y c) la tendencia sostenida al alza de esos dos indicadores.

3. Para el caso de Costa Rica, se evidencia un sensible déficit en la población graduada con algún doctorado académico, con respecto a los 15 países de la figura 2, lo cual, eventualmente, comprometería, a corto, a mediano y a largo plazo, el desarrollo académico en términos de generación, de transferencia y de aplicación de conocimiento, y en función, también, del desarrollo humano y de la competitividad. Por ende, se vería comprometida la capacidad del país para responder e integrarse al desarrollo académico mundial en esos mismos términos.

4. Con base en los datos e información de la tabla 5 , al registrarse la cantidad de población graduada con algún posgrado, en un único rubro donde no se especifica si se trata de especialidades, maestrías o doctorados, se evidencia una intensión de exponer datos generales y no específicos, lo cual se traduce en un desmérito para el doctorado académico. Según el Dr. Joaquín García Carrasco, en correo electrónico del 09 de agosto del 2019, dicho desmérito "pareciera tener su origen en la falta de cultura de doctorado académico en las universidades públicas costarricenses".

5. La conclusión anterior cobra mayor relevancia al considerar el hecho de que el doctorado académico no es, en la educación superior costarricense -incluyendo la pública-, un requisito indispensable para ascender al escalafón profesional-académico de "Catedrático o Catedrática"; incluso, dicho grado no implica, en algunos casos, ningún incentivo salarial o de otro tipo, más allá del honorífico. Tampoco supone la producción académica y generación continua de conocimiento, como requisitos para mantenerse en la categoría de "Catedrático o Catedrática".

6. Los valores de IDH e ICG, así como el sostenimiento de su tendencia al alza, para el caso costarricense, podrían estar más asociados a 
otros factores menos vinculados con la generación, la transferencia y la aplicación de conocimiento propio, como resultado del desarrollo científico y académico en programas doctorales y las investigaciones que se generen en estos. Entre esos otros factores, podría pensarse en a) alta dependencia del conocimiento foráneo para su aplicación, b) elevado gasto por endeudamiento para inversión social en aquellos rubros que modifican uno o más componentes de los indicadores IDH y ICG, sin que necesariamente se dé la generación de conocimiento autóctono, c) inversión extranjera directa asociada a factores ajenos al desarrollo científico y académico, d) economía basada en procesos productivos orientados hacia la transformación de materias primas con reducido valor agregado, más que en la generación y transferencia de conocimiento.

7. Los descriptores y resultados de aprendizaje esperados para el nivel doctoral, según el Marco de Cualificaciones para la Educación Superior Centroamericana (2013), al menos desde la perspectiva formal de educación superior, están construidos, de tal manera que las poblaciones graduadas de niveles doctorales incidan en uno o más de los componentes que integran los índices de desarrollo humano y de competitividad global, según los respectivos campos disciplinares de formación doctoral.

8. Existe consistencia y relación directa entre los conceptos: conocimiento, desarrollo humano, desarrollo de la educación, educación superior, doctorado, con respecto a los descriptores y resultados de aprendizaje esperados para el nivel doctoral, según el Marco de Cualificaciones para la Educación Superior Centroamericana (2013).

9. De acuerdo con la OCDE, para el caso de Costa Rica, el alto "gasto público en educación superior como un porcentaje del Producto Interno Bruto (PIB), incluso superior al de la mayoría de los países miembros de ese organismo" $(2017,18)$, considerando a la vez, el reciente financiamiento que el Banco Mundial otorgó al país, para fortalecer y mejorar su educación superior pública, confirma la existencia de una alta capacidad instalada en a) talento humano, b) infraestructura (física y tecnológica), c) otros recursos tangibles e intangibles, para la generación y transferencia de conocimiento.

10. En el caso de Costa Rica, la magnitud del gasto total para la educación superior pública, en términos de su PIB, y su respectivo impacto en los valores de IDH e ICG, podría estar asociado menos a la efectividad de ese gasto como inversión global, con alcance nacional, y más a factores específicos relacionados con institucionalidad, planificación, dirección y gestión, orientados a mantener el balance en la relación eficiencia-eficacia y la efectividad para a) la formación de doctores y doctoras, b) la generación y transferencia de conocimiento por parte de sus programas doctorales, y c) las investigaciones que se deriven de dichos programas. 
En otras palabras, aun cuando el gasto en educación superior pública, en términos del PID costarricense, supera el que hacen otros países de la OCDE, si no se tiene claridad en cuanto a la planificación, a la dirección y a la gestión orientadas a mantener el balance en la relación eficiencia-eficacia de ese gasto en particular, específicamente en lo que respecta a formación y desarrollo para programas doctorales, la población doctoral graduada, en el mejor de los casos, se mantendría invariable.

\section{Recomendaciones}

Para el caso costarricense, convendría:

1. Según el Dr. Joaquín García Carrasco, en su correo electrónico del 09 de agosto del 2019, con quien se departió sobre este tema, "considera que las universidades públicas costarricenses, con sus respectivos centros de investigación, aportan la mayor parte de la investigación que se realiza en el país, la promoción y el fomento del grado de doctor/a académico/a, tendría el potencial de incrementar el crecimiento de los indicadores globales de investigación en Costa Rica".

2. Profundizar aún más en la comprensión, tanto de los niveles y tipos de vinculación entre los descriptores y resultados correspondientes al nivel doctoral según el Marco de Cualificaciones para la Educación Superior Centroamericana (2013), así como en su impacto o, al menos, incidencia en aquellos factores asociados a las variaciones en los índices de IDH e ICG. No se trata solo de saber que es probable la existencia de relaciones y correlaciones en este ámbito, sino que, también, precisa fundamentar y dar sentido, desde las perspectivas, técnica, práctica y transformadora, a tales relaciones y correlaciones.

3. Diseñar los mecanismos y las dinámicas que le permitan, a la educación superior pública, un mayor monitoreo y seguimiento de las poblaciones graduadas con algún doctorado académico, así como su participación en el desarrollo humano y competitividad del país.

4. De acuerdo también con el Dr. Joaquín García Carrasco, en correo electrónico del 09 de agosto del 2019, "el actual esquema de formación doctoral, que incluye el sistema de becas para estudios de doctorado en el extranjero, ha sido efectivo, pero es costoso, extenso en el tiempo y es poco inclusivo". Esto hace que presente desbalances en la relación eficiencia-eficacia; por lo tanto, urge proponer nuevas alternativas para fomentar e implementar la formación doctoral, o al menos, para la formación de alto nivel en investigación conducente a la obtención de doctorados, en campos del saber vinculados con las agendas nacionales, regionales y mundiales de investigación, en las cuales se encuentran inmersos el desarrollo humano (IDH) y la competitividad (ICG) 
5. Dichas alternativas deben mantener balances óptimos en su efectividad y la relación eficiencia-eficacia, sobre todo, en aquellos aspectos relacionados con su duración, costo y fortalecimiento de la calidad, así como el rigor científico y académico. Preferiblemente deben ser alternativas concebidas desde una perspectiva disruptiva y compleja, como complejo es el contexto actual en el que se encontrarían inmersas.

6. Conformar los cuadros de relevo generacional, pensando en que la conformación demográfica actual ejerce una fuerte presión que acelera la tasa de jubilación o el retiro de las poblaciones que, actualmente, tienen algún doctorado académico.

7. Diseñar un esquema de incentivos, tanto para las poblaciones que decidan ingresar a un programa de formación doctoral como para aquellas poblaciones que logran graduarse de alguno de estos programas. Este esquema de incentivos debe apegarse a principios de productividad académica, racionalidad y proporcionalidad.

8. Los criterios de referencia para evaluar la calidad académica, en la educación superior universitaria, deben fortalecer los mecanismos y las dinámicas que garanticen líneas o vías de retorno entre la producción científica y académica de los programas doctorales, los otros posgrados (especialidades y maestrías), el grado y el pregrado. Con esto, se logrará que el quehacer académico mantenga su debida fundamentación en la actividad investigativa como actividad académica sustantiva.

9. Profundizar en la formulación y en el desarrollo de investigaciones sobre este tema, desde la perspectiva del pensamiento complejo, las ciencias complejas, las ciencias de la organización y la indagación sistémica manteniendo "interés práctico por comprender la problemática, enfoque sociocrítico con interés emancipador y el propósito de transformar dicha problemática" (Villalobos, 2017, 15-16).

\section{Bibliogrfía}

Alicia Gurdian-Fernández. 2007. «Método hermenéutico-dialéctico». En El paradigma cualitativo en la investigación socio-educativa, editado por Marvin Herrera Araya, 147-149. Costa Rica: Colección IDER, Coordinación Educativa y Cultural Centroamericana (CECC) de la Agencia Española de Cooperación Internacional (AECI).

BM (Banco Mundial). 2018. «Población total por país (Base de datos en formato Excel)», acceso el 5 de diciembre del 2018, https://datos.bancomundial.org/indicador/SP.POP.TOTL? name_desc=false 
British Broadcasting Corporation. 2018. «Cuáles son los 10 países con más universitarios del mundo ( $\mathrm{y}$ cuáles son los primeros de América Latina)». BBC NEWS MUNDO, 15 de agosto, https://www.bbc.com/mundo/noticias-45177236

Córdoba, Javier. 2019. «Universidades y gobierno firman acuerdo sobre FFE 2020». Semanario Universidad, 18 de julio, https://semanariouniversidad.com/ultima-hora/universidades-ygobierno-firman-acuerdo-sobre-fees-2020/

Arce Flores, Diana, Ericka Moya Vargas, Franky González Conejo, Mario Alejandro Morúa Saborío y Pablo Ramírez Solano. 2017. Estudio de seguimiento a personas graduadas de doctorado en las cuatro universidades estatales costarricenses periodo 2000-2011. San José: EUNED.

FEM (Foro Económico Mundial). 2014. «The Global Competitiveness Report 2014-2015. Suiza», acceso el 15 de julio del 2019, https://www.weforum.org/reports/global-competitiveness-report-2014$\underline{2015}$

- 2016. «The Global Competitiveness Report 2016-2017. Suiza», acceso el 15 de julio del 2019, https://www.weforum.org/reports/the-globalcompetitiveness-report-2016-2017-1FEM

- 2017. «Estos países tienen la mayor cantidad de graduados con título de doctorado», acceso el 7 de marzo del 2017, https://es.weforum.org/agenda/2017/03/estos-paises-tienen-la-mayorcantidad-de-graduados-con-titulo-de-doctorado/

Luis Eduardo González Fiegehen y Hernán Ayarza Elorza. 2016. «Calidad, evaluación institucional y acreditación en la educación superior en la región Latinoamericana y del Caribe», acceso el 1 de enero del 2016, http://biblioteca.uahurtado.cl/UJAH/Reduc/pdf/pdf/8184.pdf

Luis Ricardo Villalobos Zamora. 2017. Enfoques y diseños de investigación social: cuantitativos, cualitativos y mixtos. San José: EUNED.

Consejo Superior Universitario Centroamericano (CSUCA). Marco de cualificaciones para la educación superior centroamericana. Resultados de aprendizaje para licenciatura, maestría y doctorado. Una aproximación desde la visión académica. Guatemala: Proyecto Alfa Punete

Morúa Saborío, Mario Alejandro. (2016). «La Integración Regional de los Estudios de Posgrado Ofrecidos por las Universidades Públicas en Centroamérica y República Dominicana». Tesis doctoral. Universidad Estatal a Distancia, Costa Rica, http://repositorio.uned.ac.cr/reuned/browse? type=author\&order=ASC\&rpp=20\&value=Mor\%C3\%BAa+Sabor \%C3\%ADo\%2C+Mario+Alejandro 
Organización para la Cooperación y el Desarrollo Económico (OCDE). "Educación en Costa Rica. Aspectos destacados 2017". Acceso el 01de agosto del 2019. https://www.oecd.org/edu/school/Educacion-en-CostaRica-2017-Aspectos-Destacados.pdf

OCDE (Organización para la Cooperación y el Desarrollo Económico). 2019. «La baja en el gasto público en I+D y los riesgos proteccionistas pueden representar una amenaza para la innovación, declara la OCDE», acceso el 1 de agosto del 2019, https://www.oecd.org/centrodemexico/medios/la-baja-en-el-gastopublico-en-i-d-y-los-riesgos-proteccionistas-pueden-representar-unaamenaza-para-la-innovacion-declara-la-ocde.htm

González Casanova, Pablo. (2005). Las nuevas ciencias y las humanidades. De la academia a la política. México: Anthropos.

PNUD (Programa de las Naciones Unidas para el Desarrollo). 2019. «Índices e indicadores de desarrollo humano. Actualización estadística de 2018», acceso el 21 de mayo del 2019, hdr.undp.org/sites/default/files/2018_human_development_statistical_u pdate_es.pdf

Programa Estado de la Nación. 2019. «Quinto informe. Estado de la educación 2015», acceso el 21 de mayo del 2019, https://www.estadonacion.or.cr/educacion2015/\#inicio

- 2019. «Séptimo informe. Estado de la educación 2019», acceso el 26 de agosto del 2019, https://estadonacion.or.cr/informe/?id=b13f505f-172f42f4-b18b-f0e53d2a58d3

RAE (Real Academia Española). 2019. «Doctorado», acceso el 24 de mayo del 2019, https://dle.rae.es/?id=E3Xca2U

Unesco (United Nations Educational, Scientific and Cultural Organization) (2019). "Conocimiento». Tesauro de la UNESCO, acceso el 24 de mayo del 2019 http://vocabularies.unesco.org/browser/thesaurus/es/page/concept6288

- 2019. «Desarrollo humano». Tesauro de la UNESCO, acceso el 24 de mayo del 2019 http://vocabularies.unesco.org/browser/thesaurus/es/page/concept3619

- 2019. «Desarrollo de la educación». Tesauro de la UNESCO, acceso el 24 de mayo del 2019 http://vocabularies.unesco.org/browser/thesaurus/es/page/concept8

- 2019. «Educación superior». Tesauro de la UNESCO, acceso el 24 de mayo del 2019 http://vocabularies.unesco.org/browser/thesaurus/es/page/concept1485 